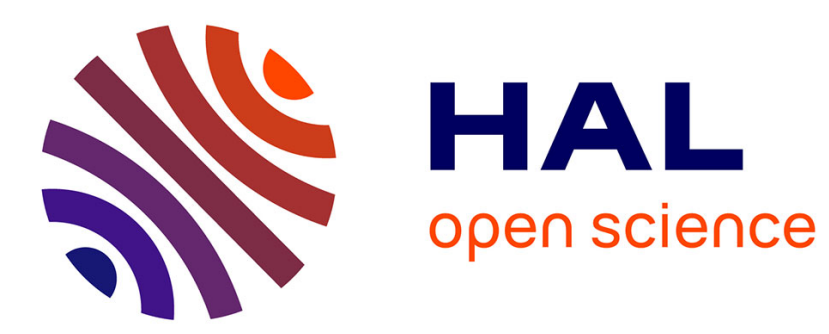

\title{
Les pratiques ludiques des adultes entre affinités électives et sociabilités familiales
}

Samuel Coavoux, David L Gerber

\section{To cite this version:}

Samuel Coavoux, David L Gerber. Les pratiques ludiques des adultes entre affinités électives et sociabilités familiales. Sociologie, 2016, 7 (2), pp.133-152. 10.3917/socio.072.0133 • halshs-01348941

\section{HAL Id: halshs-01348941 https://shs.hal.science/halshs-01348941}

Submitted on 21 Aug 2020

HAL is a multi-disciplinary open access archive for the deposit and dissemination of scientific research documents, whether they are published or not. The documents may come from teaching and research institutions in France or abroad, or from public or private research centers.
L'archive ouverte pluridisciplinaire HAL, est destinée au dépôt et à la diffusion de documents scientifiques de niveau recherche, publiés ou non, émanant des établissements d'enseignement et de recherche français ou étrangers, des laboratoires publics ou privés. 


\section{ENQUETES}

\section{Les pratiques ludiques des adultes entre affinités}

\section{électives et sociabilités familiales}

Samuel Coavoux et David Gerber (2016), « Les pratiques ludiques des adultes entre affinités électives et sociabilités familiales », Sociologie, 7(2), pp. 133-152, doi : $10.3917 /$ socio.072.0133

Dernière version auteur.

\section{Coavoux, Samuel}

Doctorant, École Normale Supérieure de Lyon, Centre Max Weber samuel.coavoux@ens-lyon.fr

\section{Gerber, David}

Doctorant, Département de sociologie, Université de Genève david.1.j.gerber@gmail.com

Cet article avance que les pratiques du jeu vidéo et celles du jeu classique sont respectivement ancrées dans des réseaux de sociabilité élective et dans des réseaux de sociabilité astreinte. Si le jeu vidéo participe de l'émancipation culturelle des enfants et adolescents (Pasquier, 2005a), le jeu classique remplit une fonction familiale : alimenter les sociabilités intergénérationnelles. L'analyse des pratiques ludiques par les sociabilités permet par ailleurs de dépasser une interprétation technologique de l'évolution des jeux. L'article s'appuie sur une enquête quantitative (par questionnaire) et qualitative (par entretien) portant sur la population adulte résidant en France en 2012. Il examine d'abord les écarts de pratiques ludiques dans le cycle de vie, discute et complète l'hypothèse d'un effet de génération, puis analyse l'ancrage de ces pratiques dans des situations de sociabilité.

âge, culture, famille, jeu, sociabilités ; age, culture, family, play, sociabilities

\section{Remerciements}

Les questionnements et les données à l'origine de cet article sont issus de la recherche collective LUDESPACE, financée par l'Agence Nationale pour la Recherche (ANR 2011 JSH 001 01, 2011-2014) et portée par Hovig Ter Minassian à l'Université François Rabelais de Tours. Les auteurs tiennent à remercier les autres participants du projet: Vincent Berry, Manuel Boutet, Isabel Colon de Carvajal, Samuel Rufat, Hovig Ter Minassian, Mathieu Triclot et Vinciane Zabban. L'ensemble des participants est responsable de la production des données utilisées, et nombre de nos analyses sont issues de discussions menées au sein de ce groupe. Les auteurs remercient également Jérôme Deauvieau et Anton Perdoncin pour leurs conseils sur les modèles statistiques, et les trois relecteurs anonymes de la revue pour leurs commentaires. David Gerber remercie en outre le Fonds national suisse de la recherche scientifique pour son soutien via une bourse de mobilité qui lui a permis de mener ces travaux. 
Nous nous proposons dans cet article d'analyser les pratiques ludiques, tant numériques que classiques, à l'aune des sociabilités familiales et électives. Notre analyse s'inscrit dans le cadre plus large de la sociologie des pratiques culturelles pour étudier le jeu domestique des adultes, un loisir rarement pris en compte par les sciences sociales. Elle vise ainsi à éclairer la diversité des fonctions sociales du jeu; en retour, l'entrée par les jeux permet plus généralement de saisir des modes de vie et des formes de sociabilité.

Notre interrogation est née, au cours d'une enquête sur la pratique du jeu vidéo en France, du constat d'un écart dans l'évolution avec l'âge de la pratique du jeu vidéo et de celle du jeu classique. Alors que le premier est fortement lié à l'enfance et à l'adolescence, et aujourd'hui, dans une moindre mesure, aux premières années de l'âge adulte, le jeu classique est plus équitablement réparti dans les classes d'âge. Il est par ailleurs plus pratiqué à l'âge adulte par les femmes que par les hommes - alors que l'inverse est vrai du jeu vidéo à tous les âges.

L'écart entre ces deux formes de jeu relève évidemment de différences générationnelles, le jeu vidéo se diffusant à mesure que les cohortes l'ayant pratiqué dès leur enfance vieillissent. Cet écart, cependant, ne ressort pas seulement d'une évolution technique. Elle ne peut agir que parce que la pratique du jeu est inscrite dans des configurations particulières de sociabilités: les relations de pairs, habituellement pensées comme électives, même si elles relèvent parfois de la "tyrannie de la majorité » (Pasquier, 2005a), et les sociabilités familiales intergénérationnelles. Le jeu classique est partagé par les générations, alors que le jeu vidéo est un des éléments de la culture jeune qui favorise l'émancipation de la sphère familiale et la reconfiguration des réseaux sociaux autour des groupes de pairs. La sociologie des pratiques culturelles rejoint ici celle de la famille.

On entend ici par jeux classiques toutes les pratiques ludiques nécessitant un matériel autre qu'informatique. La catégorie est large et recouvre les jeux institutionnalisés comme les échecs, les jeux classiques et populaires comme le loto ou la belote, ou encore les jeux dits de société, c'est-à-dire les objets ludiques «copyrighted » (Dayan 1986; cité par Fine 2015:174), produits et distribués par des sociétés commerciales, comme le Monopoly. Nous avons conscience que la distinction du « jeu vidéo » et du « jeu classique » est grossière, d'une part parce que les frontières entre les deux ne sont pas claires (bon nombre de jeux vidéo sont des adaptations informatisées de jeux existant précédemment), et d'autre part parce que le caractère « classique » des jeux que nous appelons ainsi n'est pas toujours établi. L'opposition est heuristique en ce qu'elle renvoie à des formes ludiques s'articulant autour de configurations sociales particulières. Ces configurations et leurs sociabilités sont le véritable objet de cet article, qui vise à montrer, à rebours du déterminisme technologique, que ce sont bien les formes sociales dans lesquelles prend place la pratique de ces jeux, et non leur nature technique, qui explique la différenciation de leur pratique.

L'article est structuré de la manière suivante. En partant du constat que les pratiques ludiques quotidiennes des adultes sont peu étudiées, il propose d'abord un tour d'horizon de la littérature sur les loisirs, les jeux et les sociabilités. Il décrit ensuite les données quantitatives et qualitatives ainsi que les méthodes employées dans les parties suivantes. Nous présentons dans un troisième temps les résultats des analyses de 
données quantitatives sur les déterminants des pratiques ludiques, qui nous permettent de conclure au rôle structurant des sociabilités familiales. Dans les deux dernières parties, nous affinons cette analyse en mobilisant des données issues du volet qualitatif de l'enquête pour dégager quelques propriétés des pratiques ludiques inscrites dans les sociabilités familiales et de pairs. Nous montrons que tout un pan de pratiques ludiques sont façonnées pour être en adéquation avec les situations sociales (avec qui on joue, quelles compétences sont requises) plutôt que par le seul du goût individuel. Enfin, nous revenons sur les pratiques de jeu passionnés, pour montrer que si elles sont moins ancrées dans des sociabilités astreintes, elles sont aussi tributaires de contraintes et de compromis.

\section{Jeux, loisirs et sociabilités}

Dans les études contemporaines du jeu, le jeu vidéo est largement privilégié, et le jeu classique habituellement ignoré. Cela est d'autant plus vrai que l'on se penche sur les pratiques adultes, y compris, paradoxalement, dans le champ des game studies. C'est que, plus généralement, les pratiques ludiques domestiques des adultes sont un objet rarement travaillé par les sciences sociales. Ces trois caractéristiques (pratique ludique, domestique, faite par des adultes) ne sont presque jamais réunies. De nombreux travaux portent cependant sur des objets proches, réunissant une ou deux de ces propriétés : les pratiques ludiques extra-domestiques, le jeu domestique des enfants, les autres loisirs culturels domestiques. Dans cette section, nous passons en revue ces travaux et plaidons pour une sociologie du jeu comme pratique culturelle.

La sociologie des loisirs demeure largement aujourd'hui une sociologie du temps libre. Elle s'est appuyée sur l'étude des évolutions de long terme du temps de travail professionnel comme domestique. La thèse de Joffre Dumazedier, celle d'une "civilisation du loisir» caractérisée par l'importance croissante du temps libre (Dumazedier 1962) est l'un des principaux piliers de ce champ de recherche. On y trouve l'affirmation de l'importance du loisir domestique et de sa "massification » progressive (Yonnet 1985). Cette thèse est toutefois tempérée par les nombreuses analyses des inégalités sociales d'accès aux loisirs (Chenu et Herpin 2002), qui mettent par exemple en avant l'opposition entre loisirs domestiques quotidiens dans les classes populaires et loisirs de temps long dans les classes supérieures (Coulangeon et al. 2002). Cependant, les travaux qui relèvent de cette spécialité prennent rarement en compte le jeu, comme le montrent les enquêtes de la statistique publique. Les enquêtes « Pratiques culturelles des français », dont la dernière édition incluait deux questions sur le jeu vidéo, ne se sont jamais intéressées aux autres pratiques ludiques (Donnat 2009). Les enquêtes "Emploi du temps » ne considèrent le jeu que comme éducation des enfants, bien qu'elles incluent désormais le jeu vidéo (Brousse 2015). Ces travaux nous invitent à demeurer attentifs aux différenciations sociales de la pratique des jeux, ainsi qu'à son inscription temporelle (Degenne et Lebeaux 2003), mais la sociologie du jeu comme pratique culturelle et de loisir reste à faire (Rufat et al. 2014).

Lorsque la sociologie des loisirs se saisit du jeu, c'est le plus souvent sous sa forme publique et/ou extérieure au foyer. Les jeux d'argent, en particulier les paris sportifs et les courses de chevaux, sont analysés dans leurs aspects économiques et sociaux (Digard 2001 ; Martignoni-Hutin 1997 ; Yonnet 1980). L'ethnographie des pratiques de 
jeu privilégie les réunions publiques, comme les compétitions ; ainsi par exemple du jeu d'échecs saisi par ses concours (Fine 2012; Wendling 2002). De même, la géographie des jeux de plateaux s'appuie sur les licenciés de fédérations, et concerne donc les amateurs pratiquants des jeux institués (Borzakian 2015). Si ces pratiques ne correspondent pas à l'ordinaire des jeux, l'accent mis ainsi sur les amateurs engagés peut nous aider à saisir le devenir du jeu autonome lorsqu'il persiste à l'âge adulte (voir dernière partie). À cet égard, les travaux sur le jeu de rôle, une forme de jeu de société demandant un investissement particulièrement élevé, ont été importants (Fine 1983, 2015). Cependant, ce sont, là encore, les dimensions publiques, ou du moins extra-domestiques, de la pratique du jeu de rôle qui ont été privilégiés par les sociologues, comme le jeu en club (Lizé 2004).

L'exception se trouve finalement dans une enquête portant sur l'émergence du troisième âge, où les activités de loisir des retraités incluent le jeu comme activité pouvant s'exercer à domicile, et investie surtout par les femmes (Bickel et al. 2005). L'insertion du jeu dans la famille est rarement considérée par le chercheur. La sociologie des loisirs familiaux, quant à elle, s'intéresse peu au jeu. La famille constitue pourtant le groupe le plus propice aux activités de loisirs, par sa disponibilité quotidienne. La famille est également un groupe contraignant, alors que l'activité de loisir est souvent définie par sa liberté (Harrington 2006). Kelly analyse ce paradoxe en montrant que, alors que les individus qu'il interroge dans ces enquêtes affirment vouloir des loisirs librement choisis, ils trouvent dans les loisirs familiaux semi-contraints la plus grande source de satisfaction personnelle (Kelly 1995).

Une abondante littérature sur l'enfance étudie la place du jeu dans la socialisation et l'éducation. En France, c'est dans la lignée du philosophe Jacques Henriot que se sont structurées, autour de sociologues de l'éducation, les recherches sur les jeux et les jouets (Brougère 2013). Les jouets sont un outil dans les stratégies éducatives des familles (Vincent 2000). L'observation de leur circulation dans le cadre domestique met en lumière les conflits et les négociations au sein de la fratrie, et entre parents et enfants (Dajez et Roucous 2009, 2010). Les adultes sont surtout abordés, dans ces enquêtes, par l'encadrement qu'ils produisent du jeu des enfants.

In fine, le jeu domestique des adultes a principalement été abordé sous une forme unique, celle du jeu vidéo. La naissance, entre la fin des années 1990 et le début des années 2000, d'un courant de recherche qui s'est nommé game studies ne doit pas faire illusion : la prétention à parler des jeux en général s'y heurte à la domination massive des jeux vidéo et d'autres objets numériques proches, comme les mondes virtuels, dans les travaux et les enquêtes. Par ailleurs, les études empiriques des pratiques y sont rares. Une ethnographie des mondes en ligne sensible à l'inscription matérielle des pratiques, et pas uniquement aux jeux de représentation dans le monde virtuel, s'est toutefois développée dans le monde français comme anglo-saxon (Berry 2012; Boutet 2008; Pearce 2009; Taylor 2006). Elle nous renseigne sur l'écosystème domestique du jeu vidéo (Berry 2012 ; Boutet et al. 2014), et elle insiste précisément sur le fait qu'il s'agit de «jeux d'adultes» (Boutet 2003). Quelques-uns de ces travaux étudient particulièrement l'insertion du jeu vidéo dans les relations familiales : par exemple, lorsque le don d'une console à ses parents cache l'ambiguïté des formes de la piété filiale dans la société chinoise (Lin 2014). 
La sociologie des usages a particulièrement creusé la question de la production et du maintien du lien familial et des sociabilités électives par les techniques numériques. Elles servent à maintenir une communication riche, à assurer la coordination des membres de la famille (Le Douarin 2007). Les négociations autour de l'équipement reflètent des formes d'organisations familiales, par exemple dans les familles recomposées (Pharabod 2004). Les techniques de communication sont également ce qui permet aux enfants grandissants de s'émanciper progressivement de la sphère familiale : le téléphone portable et la messagerie instantanée permettent aux parents de conserver les enfants au foyer, tout en leur laissant le loisir de nouer des liens entre pairs (Martin 2004). Ces travaux confirment et détaillent ainsi un résultat classique quant à l'évolution des sociabilités: passé l'adolescence, l'avancée dans le cycle de vie s'accompagne d'un resserrement des sociabilités autour de la famille (Forsé 1981).

L'étude des sociabilités liée aux TIC s'est étendue aux objets numériques qui ne sont pas dédiés à la communication, ainsi qu'aux pratiques culturelles. Dans un ouvrage qui a fait date, Dominique Pasquier montre ainsi l'importance des objets de la culture de masse dans la culture jeune. Les jeux vidéo constituent alors un des éléments centraux de la culture masculine, car leur pratique partagée est un moteur des dynamiques de sociabilité quotidienne au lycée (Pasquier 2005a). Plus généralement, les enquêtes montrent l'importance des sociabilités dans la détermination des pratiques culturelles, à tous les âges de la vie (Cardon et Granjon 2003; Gire et al. 2007 ; Smoreda 2002). Omar Lizardo avance ainsi que la consommation de culture de masse dans tout l'espace social s'explique par les ressources qu'elle donne aux acteurs dans leurs sociabilités quotidiennes : elle est ce dont on peut parler avec tout le monde (Lizardo 2006). Mais même les activités culturelles les plus légitimes s'insèrent dans des réseaux de sociabilités (Pasquier 2012).

Or, ce lien entre sociabilités et pratiques culturelles a une conséquence très importante pour la sociologie de la culture : il oblige à ne pas lier systématiquement l'étude du goût et celle de la pratique. Bernard Lahire pointe dans La culture des individus le fait que les pratiques réelles ne sont pas toujours le produit d'un goût personnel, mais peuvent être faites « sous influence » ou être des " pratiques d'accompagnement » (Lahire 2004, ch. 13). La musique écoutée au quotidien, par exemple, est à la fois choisie et subie : elle est soumise aux situations de travail et de loisir (DeNora 2001; Nowak et Bennet 2014). Mais même les activités culturelles demandant un engagement conséquent, comme la pratique de la musique, peuvent être partiellement déconnectées des goûts des acteurs. Vincent Dubois, Jean-Matthieu Méon et Emmanuel Pierru montrent ainsi qu'un certain nombre de musiciens d'harmonie, en particulier les plus jeunes, jouent des musiques très différentes de celles qu'ils apprécient (Dubois et al. 2010).

L'idée selon laquelle le jeu ne se résume pas à la recherche individuelle de plaisir n'est pas nouvelle. L'examen des formes historiques du jeu auquel se livre Johan Huizinga (1977, voir aussi Henricks 2010), montre que le jeu, tant chez les enfants que chez les adultes, prend des formes et remplit des fonctions sociales diverses. À un niveau individuel, la psychanalyse a investi le jeu comme une composante fondamentale du développement individuel (Winnicot, 1971). D'un point de vue macrosocial, l'émergence du sport comme jeu institutionnalisé peut être compris comme découlant d'un processus civilisationnel (Elias et Dunning 1986), ce qui fonde des perspectives 
critiques dénonçant « la pauvreté spirituelle» du jeu moderne (Duflo 2002), ou encore sa «sportification » (Bordes 2009, cité par Borzakian 2012).

\section{Données et méthode}

Cet article prend appui, empiriquement, sur l'enquête collective Ludespace, réalisée de 2011 à 2014. Nous disposons de deux principaux ensembles de données produits dans ce cadre. Il s'agit d'une part des réponses à un questionnaire sur la pratique du jeu vidéo des personnes de 11 ans et plus habitant en France $(n=2542)$, et d'autre part, d'une série d'entretiens menés avec des adultes pratiquant, occasionnellement ou régulièrement, le jeu vidéo $(\mathrm{n}=30)$.

\section{Mesurer les pratiques ludiques des Français : une enquête par questionnaire}

L'enquête par questionnaire a porté sur un échantillon représentatif de la population française, défini par la méthode des quotas. Les variables employées pour définir les quotas étaient le sexe, la profession et catégorie socioprofessionnelle, le niveau de diplôme, l'aire urbaine et la région du domicile. Deux sous-populations étaient distinguées : les plus de 18 ans $(n=2042)$ et les 11-17 ans $(n=500)$. Le questionnaire a été administré au téléphone en juin et juillet 2012 par le CREDOC. La limite d'âge inférieure a été fixée à 11 ans à partir de l'expérience des recherches en sciences de l'éducation (Scott 2008 ; Borgers et al. 2000) : il apparaissait trop difficile d'administrer un questionnaire à un enfant plus jeune, à moins de modifier considérablement les questions, et de perdre alors la comparabilité entre sous-populations. Les moins de 18 ans étaient interrogés, avec un questionnaire presque identique à celui des adultes, après que l'enquêteur ait recueilli l'accord de leurs parents, ainsi que les réponses aux questions employées pour les quotas. Dans cet article, nous nous concentrons sur le jeu des adultes. Seuls les répondants âgés de 18 ans et plus sont donc inclus dans les modélisations. Le sous-échantillon des enfants est mobilisé ponctuellement pour décrire l'évolution des pratiques avec l'âge et pour évoquer le taux de partage des pratiques ludiques avec les adultes.

Le questionnaire abordait, outre le jeu vidéo, les autres pratiques culturelles et pratiques ludiques des enquêtés, sur le modèle des enquêtes " pratiques culturelles des Français » (Donnat 2009). Nous examinons dans cet article les seules pratiques ludiques, classiques ou vidéo. Nous employons donc les variables décrivant l'étendue des genres de jeux classiques et de jeux vidéo pratiqués. La formulation des questions portant sur les genres était la même pour les deux ensembles de jeux. Pour le jeu classique, nous demandions aux enquêtés s'ils avaient joué au moins une fois au cours des douze derniers mois à chacun des 6 genres proposés (jeux de construction; jeux de loto, puzzle ou domino; jeux de société ; jeux de stratégie, échecs ou backgammon; jeux de cartes; jeux de rôle, de figurines ou de cartes à collectionner). Nous avons, dans cette enquête, adopté une définition large du « jeu vidéo », incluant l'ensemble des dispositifs ludiques présents sur des terminaux électroniques. Sont donc concernés aussi bien les jeux de patience installés par défaut sur certains systèmes d'exploitation (par exemple le jeu de cartes Freecell sur Windows) que les jeux le plus souvent associés aux jeux vidéo (jeux de tir en vue subjective, jeux de stratégie en temps réel, etc.), et ce sur tout type de 
supports (du PC aux consoles en passant par les smartphones, consoles portables et autres tablettes). Les jeux vidéo étaient saisis à travers 19 genres (du jeu préinstallé au jeu de stratégie en temps réel, en passant par le jeu de cartes, le jeu de musique et de danse, ou encore le jeu de course ${ }^{1}$ ). D'autres questions permettaient de qualifier cette pratique (fréquence, terminaux, lieux du jeu, compagnons de jeu, etc.).

\section{Analyses statistiques}

Dans cet article, nous comparons l'évolution de la pratique du jeu vidéo et des jeux classiques avec l'âge, et testons l'hypothèse selon laquelle la configuration du réseau social, et en particulier sa reconfiguration au cours du cycle de vie, influe fortement sur ces pratiques. Nous construisons à partir du questionnaire deux variables synthétiques de pratique et d'intensité de la pratique pour chacun des deux ensembles de jeux (soit quatre variables). La variable de pratique est dichotomique : la variable pratique du jeu vidéo (resp. jeu classique) vaut 1 si la personne a joué au moins une fois au cours des douze derniers mois à un jeu vidéo (resp. jeu classique), et 0 sinon. La variable d'intensité est un score composé du nombre de genres de jeux vidéo (resp. classiques) différents pratiqués au cours des douze derniers mois. Elle s'étale de 0 à 19 pour les jeux vidéo et de 0 à 6 pour les jeux classiques. Nous employons d'abord des statistiques descriptives et des modèles linéaires croisant intensité et pratique avec l'âge des enquêtés.

Dans les analyses suivantes, nous employons également les variables dichotomiques de genre de jeu pratiqué. Nous modélisons, par des régressions logistiques la probabilité d'avoir joué à un jeu vidéo, à un jeu classique, à chacun des dix-neuf genres de jeux vidéo et à chacun des six genres de jeux classiques au cours des douze derniers mois. L'ajout de modèles de genres permet de qualifier les différences qui existent à l'intérieur de chacune des catégories : certains genres de jeu vidéo, comme le jeu éducatif, sont structurés sur le modèle des jeux classiques.

Les modèles logit sont affectés par un problème d'hétérogénéité inobservée qui rend problématique la comparaison des coefficients, comme le montre Carina Mood (2010). Pour contrôler le bien-fondé de notre argument, nous avons donc vérifié systématiquement par des modèles de probabilité linéaire (Linear Probability Model $[\mathrm{LPM}]^{2}$ ) les coefficients issus des modèles logit, suivant en cela ses conseils (Mood 2010, p. 78-79). Nous ne commentons que les résultats validés par les deux méthodes. Nous présentons dans le corps de l'article les résultats de quelques modèles logit (dont les sociologues sont plus familiers que les LPM), par souci d'économie. L'intégralité des résultats est disponible en annexe.

\footnotetext{
${ }^{1}$ Pour la passation, cette liste était présentée en trois temps, trois listes de six genres proposés successivement, à la condition d'une réponse positive à une question filtre ( "Avez-vous joué à d'autres jeux vidéo que ceux que j'ai cité ? »)

2 Il s’agit de régressions linéaires classiques, par la méthode des moindres carrés observés, appliquées à une variable dichotomique.
} 
Les analyses ont pour objectif de démêler les effets de l'âge et de la configuration familiale. Les limites matérielles du questionnaire ne nous ont pas permis d'obtenir une vue détaillée des configurations familiales, et nous avons privilégié, dans le recueil des données, les variables portant sur la composition du foyer. C'est donc à partir de la présence d'enfants au foyer et de leur âge que nous mesurons la configuration familiale. Trois cas sont distingués : l'absence d'enfants au foyer, la présence d'au moins un enfant de moins de 10 ans, la présence d'enfants dont le plus jeune a au moins 11 ans. Puisque nous cherchons à mesurer l'effet de la configuration familiale à âge constant, l'âge constitue la variable de contrôle la plus importante. Enfin, nous contrôlons dans les modèles l'effet de la position sociale (mesurée par la PCS de l'enquêté), du sexe, et du niveau de diplôme (en trois modalités : inférieur, égal, ou supérieur au baccalauréat). Nous avons également contrôlé l'effet du revenu du ménage, qui ne modifiait pas les résultats généraux. Étant donné que $12 \%$ des enquêtés adultes n'ont pas renseigné cette variable (refus de répondre ou « ne sait pas »), nous avons préféré retirer cette variable des modèles finaux pour ne pas limiter la base des répondants analysée dans les modèles.

\section{L'enquête par entretien}

Notre deuxième corpus de données est constitué d'entretiens qui ont servi à détailler les modalités des pratiques ludiques au regard des biographies familiales, professionnelles, scolaires et culturelles. Les personnes interviewées ont été recrutées sur la base d'un fichier d'adresses issu de l'enquête téléphonique décrite plus haut. Nous avons donc pu sélectionner des profils variés et contrastés, à la fois par leurs propriétés sociales et par leurs pratiques ludiques. En raison du peu de connaissances sur les modalités ordinaires des pratiques ludiques d'adultes (contrairement aux pratiques enfantines ou à celles d'adultes passionnés) seuls des joueurs de plus de 18 ans ont été interviewés. Il a en outre fallu recourir à des enquêtés recrutés par effet «boule de neige » dans l'entourage personnel et professionnel des membres du collectif de la recherche, en particulier pour accéder à des joueurs disposés à parler de pratiques sans appétence.

Les entretiens, d'une durée moyenne d'une heure vingt, ont été menés en face à face, pour la plupart au domicile des enquêtés. Nous avons employé un guide d'entretien faiblement directif, mais qui abordait systématiquement les dimensions extra-ludiques des biographies individuelles (trajectoire scolaire, professionnelle, et familiale). Il demandait aux enquêtés de décrire en détail leurs pratiques ludiques actuelles - avec qui on joue, à quoi, comment, quand et où -, mais aussi de reconstituer leur trajectoire biographique et d'y replacer leurs pratiques passées. Le fait de mener ces entretiens au domicile de l'enquêté permettait de creuser les modalités concrètes des pratiques domestiques du fait de la proximité des lieux et matériaux de jeu : collections de jeux, dispositifs audiovisuels et informatiques, etc. Si les jeux vidéo constituaient la focale principale du guide, celui-ci incitait aussi à demander quels jeux non numériques étaient pratiqués. L'orientation première de l'enquête sur les jeux vidéo plutôt que les jeux en général constitue la limite la plus évidente de ces données - nous ne disposons pas du même degré de détail sur les pratiques classiques que sur les pratiques numériques.

L'ensemble de ces entretiens a été transcrit et soumis à une analyse de contenu qualitative. D'abord, un découpage thématique (dans le logiciel Sonal) a servi à identifier les passages concernant le jeu dans le cadre familial et les passages où étaient 
abordés les goûts en matière de jeux. Ensuite, des analyses plus ciblées ont été menées en codant (avec le logiciel Atlas.ti) les formes d'engagement et les configurations sociales qui apparaissaient dans les expériences de jeu décrites. L'ensemble de ces passages a permis de saisir des modalités variables d'engagement dans les jeux ainsi que l'expression des goûts ou dégoûts de l'enquêté à leur égard.

\section{Âge, configuration familiale et pratiques ludiques}

L'analyse des déterminants sociaux de la pratique ludique révèle deux différences importantes entre les jeux numériques et les jeux classiques: la sensibilité plus importante de ces derniers à la configuration familiale, et l'effet conservateur sur les pratiques ludiques classiques de la présence au foyer de jeunes enfants. Chez les adultes, le jeu classique est d'autant plus fréquent et intense que sont présents au foyer des enfants en bas âge, et la pratique partagée avec les enfants est importante. Il n'en va pas de même pour le jeu vidéo. Alors que le jeu classique rattache les enfants au foyer, le jeu vidéo appartient à l'ensemble des produits culturels qui participent de l'affirmation de leur autonomie, et reconfigure leurs loisirs en direction des pairs. Nous extrapolons ici cette thèse soutenue par Dominique Pasquier (2005a) pour affirmer que, à l'âge adulte, la pratique des jeux vidéo demeure ancrée dans des sociabilités électives, alors que la pratique des jeux classiques est extrêmement liée à la configuration familiale.

\section{Âge, sexe et pratique du jeu}

Les enquêtes Pratiques culturelles des Français, dans son volet de 2007, et Ludespace convergent pour montrer les liens de la pratique du jeu vidéo avec l'âge, puis le sexe et la position sociale, mesurée par la catégorie socio-professionnelle, le diplôme ou le revenu (Rufat et al. 2014), que l'on mesure l'intensité ou la diversité de la pratique. L'âge est donc la variable socio-démographique ayant le plus fort effet. Le taux de pratique (avoir joué aux jeux vidéo au cours des douze derniers mois) décroît linéairement à mesure que l'on avance en âge (fig. 1). Alors que les enfants et adolescents jouent dans leur quasi-totalité (plus de 95\%, filles et garçons compris), moins de 30\% des plus de 60 ans sont concernés. L'intensité de la pratique est également une fonction linéaire décroissante de l'âge (si l'on mesure l'intensité de la pratique au nombre de genres de jeux vidéo différents pratiqués, un score allant de 0 à $19 ; \mathrm{r}=-0,6)$. 


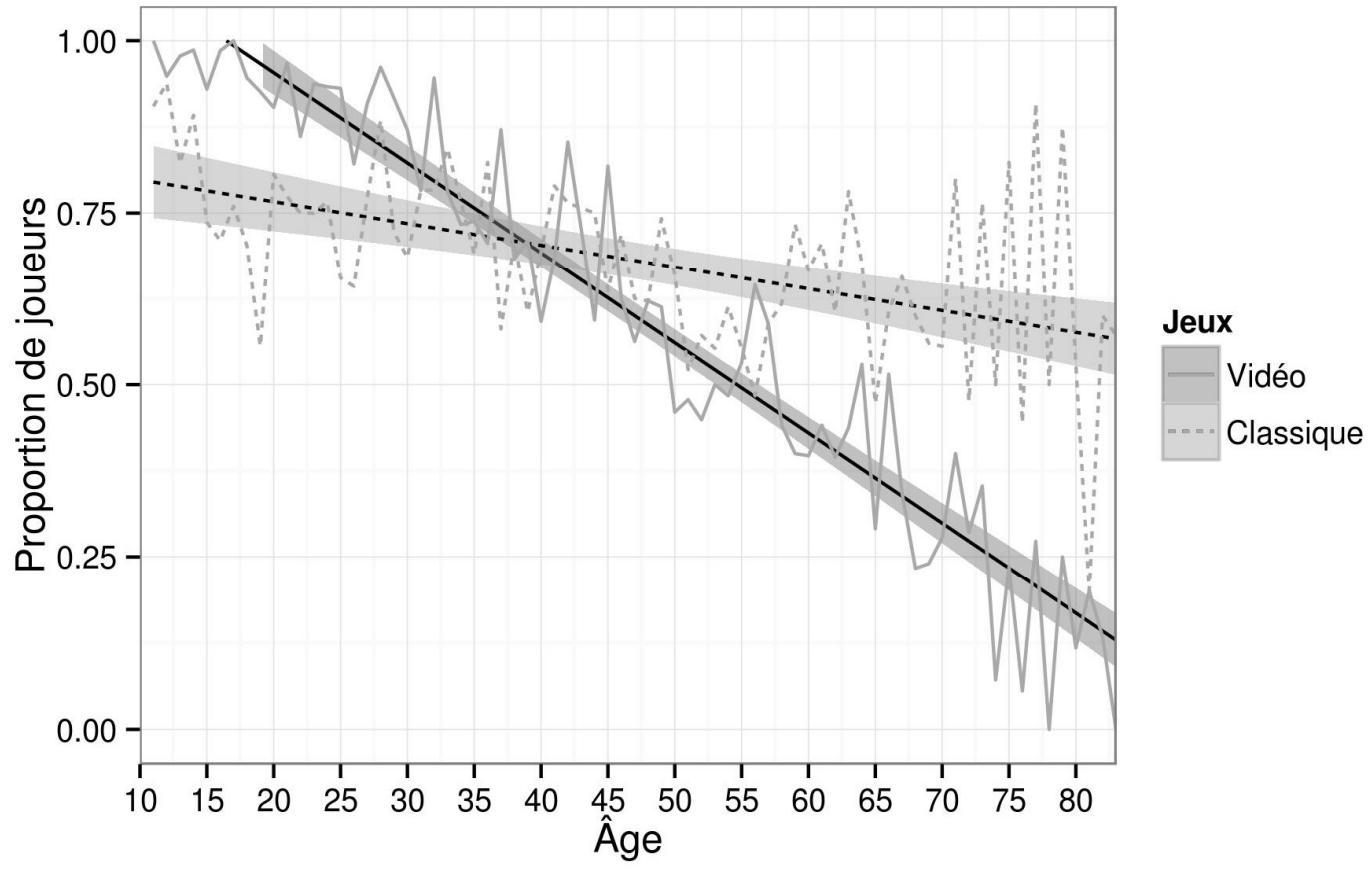

Figure 1 : Proportion de joueurs par âge

Source: enquête Ludespace (2012). $\mathrm{n}=2542$. Les droites représentent les valeurs de la proportion de joueurs (resp. de jeux vidéo $\left[\mathrm{R}^{2}=0,93\right]$ et de jeux classiques $\left[\mathrm{R}^{2}=0,2\right]$ ) prédites par des modèles de régression linéaire, et peuvent être lues comme de la tendance générale de ces variables. L'erreur standard de ces prédictions est représentée par les rubans gris.

La pratique des jeux classiques décroît elle aussi avec l'âge, mais en suivant une pente plus douce et plus irrégulière que celle des jeux vidéo. Alors que, chez les enfants et les adolescents, le jeu vidéo domine le jeu classique, en particulier chez les 14-17 ans, le déclin plus rapide du jeu vidéo est tel que les deux courbes se croisent autour de 40 ans. Le jeu vidéo est plus répandu en-deçà de cet âge ; au-delà, c'est le jeu classique qui domine. Celui-ci rassemble toujours au moins $60 \%$ d'une classe d'âge. Comme pour la proportion de joueurs, l'association de l'âge avec l'intensité de pratique du jeu classique est moins intensive que pour le jeu vidéo (on la mesure, là encore, par un score de 0 à 6 , comptabilisant le nombre de genres de jeux classiques pratiqués au cours des douze derniers mois; $r=-0,23)$. La relative stabilité de la courbe des jeux classiques et l'importance de la pente pour la courbe des jeux vidéo se lit dans la différence d'ajustement des droites de régression sur la fig. 1.

Chez les adultes, les femmes sont légèrement plus nombreuses que les hommes à avoir joué à au moins un jeu classique au cours des 12 derniers mois (69\% contre 64\%) alors qu'elles sont bien moins nombreuses dans le cas du jeu vidéo (53\% contre $66 \%$ ). Les hommes pratiquent significativement plus de genres de jeux vidéo que les femmes en moyenne (3,5 contre 2,1 sur 19 genres possibles $\left.{ }^{3}\right)$, et les femmes significativement plus

3 Tous les résultats d'analyses statistiques de l'enquête par questionnaire cités dans 
de genres de jeux classiques que les hommes en moyenne (2,6 contre 2,3 sur 6 genres possibles).

La configuration familiale influe également sur les pratiques de jeu. Les adultes vivant dans un foyer où vit également un enfant de 10 ans ou moins sont $75 \%$ à avoir joué à un jeu classique au cours des douze derniers mois (contre $67 \%$ de la population), et autant à avoir joué à un jeu vidéo au cours des douze derniers mois (contre 59\% de la population). Ils jouent à des jeux classiques plus variés $(3,4$ genres contre 2,4 en moyenne) et à des jeux vidéo plus variés (4,1 genres contre 2,8 en moyenne). Ils sont également plus jeunes (37 ans en moyenne, contre 48 ans pour la population). Les modélisations permettent alors de démêler l'effet de ces différentes variables explicatives sur la pratique du jeu.

\section{Modéliser l'effet de la configuration familiale sur la pratique du jeu}

Les modèles prennent pour variable dépendante la pratique d'un genre de jeu, classique ou numérique. Est modélisée la probabilité d'avoir, au cours des douze derniers mois, pratiqué le jeu vidéo, le jeu classique, et des genres particuliers de jeu. Les variables indépendantes sont les mêmes dans tous les modèles : le sexe (modalité de référence : homme), la profession et catégorie socioprofessionnelle de l'enquêté (modalité de référence : employés), le niveau de diplôme (modalité de référence : baccalauréat), l'âge (en années), et la présence d'enfants au foyer (modalité de référence : pas d'enfants). Comme indiqué dans la section décrivant la méthode, nous ne commentons ici que les résultats confirmés par les deux méthodes de modélisation, logit et LPM.

L'âge constitue le meilleur prédicteur de chacune des pratiques, qu'il s'agisse de la pratique globale ou de genres en particulier. Son effet est toujours significatif et négatif : chaque année supplémentaire diminue la probabilité de jouer à l'ensemble des genres de jeux, classiques comme vidéo. L'âge a donc bien un effet propre, toutes variables du modèle tenues égales par ailleurs.

L'effet de la configuration familiale est plus contrasté. Toutes variables du modèle tenues égales par ailleurs, le fait de vivre dans un foyer avec des enfants de moins de 10 ans accroit les chances de pratiquer un jeu classique. L'effet se ressent sur tous les genres de jeux classiques, sauf les jeux de cartes. Il est particulièrement fort sur les jeux de construction (les adultes vivant avec un jeune enfant sont 4,5 fois plus susceptibles de pratiquer un tel genre que ceux qui n'ont pas d'enfant à la maison), mais aussi de loto, puzzle, domino ( 3 fois plus), et de société ( 2 fois plus). Le fait d'avoir un enfant de 11 ans et plus diminue par contre les chances d'avoir pratiqué un jeu de construction ou de stratégie. La configuration familiale, par contre, ne présente pas d'effet significatif sur la pratique du jeu vidéo lorsque l'on contrôle par les variables du modèle. Lorsque l'on étudie les genres un à un, on constate que le même effet positif des enfants de moins de 10 ans s'applique aux jeux vidéo éducatifs, ainsi qu'aux jeux d'adresse. La majeure partie des autres genres n'est pas affectée. Enfin, les modèles offrent des

l’article sont développés dans l’annexe électronique. 
résultats contradictoires sur quelques genres de jeu vidéo. Dans plusieurs cas, les modèles logit trouvent un effet positif des enfants de moins de 10 ans sur la pratique du genre, que le modèle LPM ne confirme pas, et qui pourrait donc provenir des limites du modèle. Enfin, lorsque le modèle LPM montre un effet que le modèle logit ne confirme pas, cet effet est négatif : ainsi des jeux de tirs (FPS) et des jeux de simulation de stratégie militaire (wargame).

L'effet propre du sexe est également fort. Il est positif sur les jeux classique, et négatif sur les jeux vidéo (les femmes ont une probabilité 1,6 fois plus importante de ne pas jouer que les hommes, toutes variables du modèle tenues égales par ailleurs). On retrouve ces effets sur de nombreux genres. Parmi les jeux classiques, les femmes sont plus susceptibles que les hommes de jouer aux jeux de construction, de loto, de société, et de rôle, mais moins susceptibles de pratiquer des jeux de stratégie. En ce qui concerne le jeu vidéo, seuls les jeux de simulation de vie (comme Les Sims) sont plus probablement pratiqués par des femmes que des hommes; la plupart des autres genres sont moins susceptibles d'être pratiqués par les femmes, qui sont par exemple 12 fois plus susceptibles que les hommes de ne pas avoir joué à un jeu de tir au cours des douze derniers mois.

La position sociale affecte également certaines des pratiques ludiques. Toutes variables du modèle tenues égales par ailleurs, les ouvriers sont moins susceptibles de jouer à des jeux classiques comme à des jeux vidéo, en particulier aux jeux de construction, de société, et de stratégie (jeux classiques), et aux jeux préinstallés (dont la pratique est liée à l'usage professionnel de l'informatique [Cézard 2000]), de chiffres et de lettres, éducatifs, d'adresse et de sport (jeux vidéo). Les cadres sont eux plus susceptibles de jouer à des jeux classiques de cartes. À cela s'ajoute le diplôme, qui a un effet positif sur le jeu classique en général, sur les jeux classiques de cartes, de société, et de stratégie, et sur le jeu vidéo des chiffres et de lettres, d'adresse, de sport, et de simulation militaire. 


\begin{tabular}{|c|c|c|c|c|c|}
\hline & \multicolumn{5}{|c|}{ Variable modélisée } \\
\hline & \multicolumn{2}{|c|}{ Jeu classique } & \multicolumn{3}{|c|}{ Jeux vidéo } \\
\hline & $\begin{array}{l}\text { Jeu } \\
\text { classique } \\
(1)\end{array}$ & $\begin{array}{l}\text { Construction } \\
(2)\end{array}$ & $\begin{array}{l}\text { Jeu vidéo } \\
\text { (3) }\end{array}$ & $\begin{array}{l}\text { Éducatif } \\
\text { (4) }\end{array}$ & $\begin{array}{l}\text { FPS } \\
(5)\end{array}$ \\
\hline \multicolumn{6}{|l|}{$\begin{array}{l}\text { Configuration } \\
\text { familiale }\end{array}$} \\
\hline Pas d'enfant & ref. & ref. & ref. & ref. & ref. \\
\hline Enfant $0-10$ ans & $0.326^{* *}$ & $1.515^{* * *}$ & -0.069 & $0.775^{* * *}$ & -0.115 \\
\hline Erreur type & $(0.134)$ & $(0.129)$ & $(0.147)$ & $(0.137)$ & $(0.201)$ \\
\hline Odds-ratio & 1.385 & 4.550 & 0.933 & 2.171 & 0.892 \\
\hline Enfant $11+$ ans & -0.114 & $-0.254^{*}$ & -0.083 & -0.001 & -0.056 \\
\hline Erreur type & $(0.125)$ & $(0.130)$ & $(0.129)$ & $(0.191)$ & $(0.337)$ \\
\hline Odds-ratio & 0.892 & 0.775 & 0.921 & 0.999 & 0.946 \\
\hline Âge (années) & $-0.010^{* * *}$ & $-0.010^{* * *}$ & $-0.069^{* * *}$ & $-0.046^{* * *}$ & $-0.107^{* * *}$ \\
\hline Erreur type & $(0.003)$ & $(0.003)$ & $(0.004)$ & $(0.005)$ & $(0.010)$ \\
\hline Odds-ratio & 0.990 & 0.990 & 0.934 & 0.955 & 0.899 \\
\hline \multicolumn{6}{|l|}{ Sexe } \\
\hline Homme & ref. & ref. & ref. & ref. & ref. \\
\hline Femme & $0.195^{*}$ & $0.367^{* * *}$ & $-0.459^{* * *}$ & 0.092 & $-2.524^{* * *}$ \\
\hline Erreur type & $(0.109)$ & $(0.108)$ & $(0.117)$ & $(0.134)$ & $(0.254)$ \\
\hline Odds-ratio & 1.216 & 1.444 & 0.632 & 1.097 & 0.080 \\
\hline \multicolumn{6}{|l|}{ PCS } \\
\hline $\begin{array}{l}\text { Agriculteurs } \\
\text { exploitants }\end{array}$ & -0.298 & 0.324 & -0.457 & -0.377 & -0.188 \\
\hline Erreur type & $(0.363)$ & $(0.373)$ & $(0.397)$ & $(0.578)$ & $(0.862)$ \\
\hline Odds-ratio & 0.742 & 1.383 & 0.633 & 0.686 & 0.829 \\
\hline $\begin{array}{l}\text { Artisans, } \\
\text { commerçants et } \\
\text { chefs d'entreprise }\end{array}$ & -0.322 & $-0.464^{*}$ & -0.298 & 0.021 & -0.289 \\
\hline Erreur type & $(0.234)$ & $(0.258)$ & $(0.257)$ & $(0.318)$ & $(0.492)$ \\
\hline Odds-ratio & 0.724 & 0.629 & 0.742 & 1.021 & 0.749 \\
\hline $\begin{array}{l}\text { Cadres et } \\
\text { professions } \\
\text { intellectuelles } \\
\text { supérieures }\end{array}$ & 0.013 & 0.224 & -0.113 & -0.093 & 0.056 \\
\hline Erreur type & $(0.188)$ & $(0.180)$ & $(0.192)$ & $(0.232)$ & $(0.357)$ \\
\hline Odds-ratio & 1.013 & 1.251 & 0.893 & 0.911 & 1.058 \\
\hline
\end{tabular}




\begin{tabular}{|c|c|c|c|c|c|}
\hline $\begin{array}{l}\text { Professions } \\
\text { Intermédiaires }\end{array}$ & 0.109 & 0.129 & -0.048 & -0.056 & -0.165 \\
\hline Erreur type & $(0.160)$ & $(0.154)$ & $(0.166)$ & $(0.193)$ & $(0.311)$ \\
\hline Odds-ratio & 1.115 & 1.137 & 0.953 & 0.946 & 0.848 \\
\hline Employés & ref. & ref. & ref. & ref. & ref. \\
\hline Ouvriers & $-0.417^{* * *}$ & $-0.530^{* * * *}$ & $-0.451^{* *}$ & $-0.370^{*}$ & 0.146 \\
\hline Erreur type & $(0.161)$ & $(0.172)$ & $(0.179)$ & $(0.214)$ & $(0.292)$ \\
\hline Odds-ratio & 0.659 & 0.589 & 0.637 & 0.691 & 1.157 \\
\hline Étudiants & -0.323 & 0.078 & -0.219 & -0.493 & $-0.661^{*}$ \\
\hline Erreur type & $(0.288)$ & $(0.270)$ & $(0.415)$ & $(0.304)$ & $(0.387)$ \\
\hline Odds-ratio & 0.724 & 1.081 & 0.803 & 0.611 & 0.516 \\
\hline $\begin{array}{l}\text { Autres personnes } \\
\text { sans activité } \\
\text { professionnelle }\end{array}$ & -0.233 & -0.054 & -0.240 & -0.161 & $-1.010^{* * *}$ \\
\hline Erreur type & $(0.182)$ & $(0.182)$ & $(0.205)$ & $(0.217)$ & $(0.370)$ \\
\hline Odds-ratio & 0.792 & 0.948 & 0.787 & 0.851 & 0.364 \\
\hline \multicolumn{6}{|l|}{ Diplôme } \\
\hline Moins que bac & $-0.435^{* * *}$ & -0.125 & -0.171 & -0.192 & -0.354 \\
\hline Erreur type & $(0.135)$ & $(0.138)$ & $(0.148)$ & $(0.173)$ & $(0.241)$ \\
\hline Odds-ratio & 0.647 & 0.883 & 0.843 & 0.825 & 0.702 \\
\hline $\mathrm{Bac}$ & ref. & ref. & ref. & ref. & ref. \\
\hline Plus que bac & $0.401^{* * *}$ & 0.176 & 0.012 & 0.160 & -0.141 \\
\hline Erreur type & $(0.146)$ & $(0.138)$ & $(0.155)$ & $(0.167)$ & $(0.233)$ \\
\hline Odds-ratio & 1.494 & 1.193 & 1.012 & 1.173 & 0.868 \\
\hline Intercept & $1.195^{* * *}$ & -0.327 & $4.294^{* * *}$ & 0.361 & $3.094^{* * *}$ \\
\hline Observations & 2,022 & 2,022 & 2,022 & 2,022 & 2,022 \\
\hline Akaike Inf. Crit. & $2,468.4$ & $2,483.5$ & $2,213.5$ & $1,732.4$ & 915.4 \\
\hline Note: & \multicolumn{5}{|c|}{$p<0.1 ; \boldsymbol{p}<0.05 ; \mathrm{p}<0.01$} \\
\hline
\end{tabular}

Tableau: Modélisations (logit) de la probabilité de jouer à cinq genres de jeux classiques et vidéo.

Lecture: On trouve dans chaque case le coefficient, l'erreur standard, et l'odds-ratio. Toutes variables du modèle tenues égales par ailleurs, les adultes vivant avec un enfant de moins de dix ont 4,38 fois plus de chances d'avoir pratiqué un jeu de construction au moins une fois au cours des douze derniers mois. Lorsqu'un odds ratio est inférieur à 1 , il est possible de le lire en prenant son inverse. Les ouvriers sont $1 / 0,589=1,7$ fois plus susceptibles que les employés de ne pas avoir joué à un jeu de construction au cours des douze derniers mois.

La présence d'enfants au foyer ne se fait donc sentir que sur les jeux classiques, et sur quelques genres de jeux vidéo. Il s'agit à chaque fois des pratiques les plus susceptibles d'usages éducationnels ou récréatifs familiaux et partagés : jeux de société, jeux de construction, jeux vidéo éducatifs et d'entraînement cérébral, et jeux d'adresse, 
autorisant souvent une pratique partagée. La probabilité accrue des pratiques de jeux classiques des femmes peut alors être liée à leur plus fort investissement dans le soin donné aux enfants (Brousse 2015). Le jeu classique, à l'exception du jeu de cartes, apparait d'abord lié à la sociabilité intergénérationnelle, contrairement à la plupart des jeux vidéo. C'est ce que confirment les chiffres portant sur les partenaires du jeu. Ainsi, en ce qui concerne le jeu vidéo, seuls $36 \%$ des adultes joueurs et $21 \%$ du total des adultes interrogés ont joué au moins une fois avec un enfant durant l'année écoulée. À l'inverse, $69 \%$ des adultes interrogés ont joué avec un enfant à un jeu classique durant la même période, et seuls $13 \%$ d'entre eux interrogés ont joué à un jeu classique durant l'année, mais ne l'ont jamais fait avec un enfant. Le jeu vidéo est, chez deux tiers des adultes au moins, inscrit dans d'autres relations que la parenté. D'ailleurs, chez les enfants également, les parents n'apparaissent pas comme un partenaire privilégié du jeu vidéo : ils sont loin derrière les frères et sœurs, ainsi que les amis. Durant l'enfance et l'adolescence, le jeu vidéo est prioritairement associé aux sociabilités affinitaires (Pasquier 2005a), ce qui explique sa prévalence aussi importante - la quasi-totalité des 11-17 ans, garçons comme filles, joue au moins occasionnellement. Nous montrons que ce constat peut être étendu à l'âge adulte : si le jeu vidéo est alors moins important, il n'en reste pas moins associé à des relations affinitaires plutôt qu'à la famille.

Le déclin rapide du jeu vidéo provient d'une part des différences de socialisation entre les cohortes, qui produit des écarts d'appétence et de compétence, et d'autre part de ce que les fondements culturels de la sociabilité se reconfigurent à l'âge adulte, au profit, notamment, de la télévision (Boullier 2004), d'Internet (Granjon et Le Foulgoc 2010) et des autres médias. Les objets culturels supportant la sociabilité quotidienne se renouvellent après 18 ans. Le jeu vidéo appartient donc à un répertoire culturel très fort à l'adolescence, mais qui décline avec l'avancée en âge. La lenteur du déclin de la pratique du jeu classique avec l'âge ainsi que la surreprésentation des femmes parmi les pratiquants de ces jeux, étonnante par rapport à la faible féminisation des joueurs de jeux vidéo, s'expliquent alors par le fait que ces pratiques sont inscrites dans l'éducation des enfants.

\section{Évolutions biographiques et transmissions intergénérationnelles du jeu}

L'inscription différenciée du jeu classique et du jeu vidéo dans les sociabilités se perçoit à l'échelle individuelle dans l'évolution biographique des pratiques que permettent de saisir les entretiens, contrairement au questionnaire. Les récits que font les enquêtés de leurs pratiques mettent en lumière l'association du jeu classique aux sociabilités familiales intergénérationnelles, dans leur enfance avec leurs aînés, comme à l'âge adulte, avec leurs enfants. On trouve également dans les récits des hommes de moins de quarante ans, les plus concernés par le jeu vidéo, des descriptions de l'autonomisation culturelle vis-à-vis de la famille à travers la pratique du jeu vidéo entre pairs.

Le jeu classique demeure fortement associé aux configurations familiales par nos enquêtés : Lego et Memory avec les parents, belote et Scrabble avec les grands-parents, jeux de plateau comme le Monopoly, La bonne paye et le Trivial Pursuit, qui se jouent en famille et qui sont occasionnellement maintenus à l'âge adulte lors de week-ends et réunions de famille élargie. Les pratiques s'inscrivent dans des rythmes familiaux; 
certaines renvoient à des moments de la semaine voire de l'année (vacances, week-ends ou mercredi pour les enfants). Les types de jeux pratiqués sont variés, car les pratiques s'adaptent aux situations de sociabilité, au nombre, à l'âge, et aux intérêts des différents participants. Une enquêtée (Amélie, F, 29 ans, enseignante, en couple, sans enfants) évoque aussi bien le Memory (le mercredi avec sa grand-mère et des cousins plus jeunes) que les jeux des cartes classiques (belote, rami, crapette), joués seulement avec les grands-parents.

La carrière ludique classique de nos enquêtés - la suite de jeux pratiqués - s'interrompt souvent à l'adolescence. Les souvenirs de jeux classiques sont le plus souvent liés soit à l'enfance, soit aux sociabilités familiales extraordinaires (vacances et fêtes familiales) à l'adolescence. C'est que la pratique du jeu fait alors place à d'autres loisirs plus personnalisés et liés au groupe de pairs (Octobre et al. 2010). Chez les hommes de moins de 40 ans, une illustration fréquente de cette transition consiste en la mutation des pratiques de jeu classique en pratique du jeu vidéo: «quand j'avais vraiment des copains pour jouer, on a passé plus sur du jeu vidéo » $(\mathrm{H}, 33$, cadre en informatique, en couple avec un enfant). Les jeux vidéo semblent bien présents avant l'adolescence, mais ils sont alors, comme les jeux traditionnels, surtout pratiqués dans la fratrie (Dajez et Roucous 2010). Ces pratiques connaissent leur apogée à l'adolescence, puis tendent à diminuer avec l'entrée dans la vie active, la cohabitation, et la parentalité. Elles peuvent cependant durer un certain temps. Mireille (F, 38 ans, chargée d'étude, mariée, deux enfants) adopte le jeu vidéo après sa rencontre avec son mari, et joue fréquemment avec lui à la console, avant qu'ils abandonnent tous deux cette pratique lorsqu'arrivent leurs enfants.

Les pratiques du jeu classique qui s'amenuisent durant l'adolescence reviennent cependant à l'âge adulte. En effet, la longévité des jeux classiques constitue l'une de leurs dimensions les plus importantes: c'est parce que les parents d'aujourd'hui disposent de produits similaires, voire identiques à ceux de leur enfance qu'ils peuvent prendre des repères dans un répertoire familial constitué au moins depuis lors (Vincent 2001). Le jeu constitue alors un repère pour l'éducation.

Davantage que le jeu vidéo, le jeu classique accompagne les sociabilités intergénérationnelles. Il est ce qui rassemble enfants, parents, et grands-parents. Ça n'est pas le cas, aujourd'hui du moins, pour les jeux vidéo, qui n'a pas, même pour les générations adultes ayant connu sa massification durant leur enfance, d'ancrage dans les traditions familiales. Au contraire, les pratiques sont peu accompagnées par les parents, voire prises dans des conflits entre parents et enfants. L'évolution importante des techniques et des supports n'a pas permis de stabiliser des formes de jeux aussi durables que les jeux classiques.

\section{Le jeu dans les sociabilités familiales}

Si le jeu est souvent perçu comme une activité passionnée, voire pathologique, ni Le Joueur de Dostoïevski ni les férus de jeux vidéo ne constituent l'ordinaire des pratiques ludiques. D'une part, le jeu fait rarement l'objet d'un engagement intense. D'autre part, sa pratique s'inscrit moins dans une logique de préférences individuelles que dans des situations de sociabilités. Ce serait donc une erreur pour la sociologie de la culture de considérer que le goût et la pratique sont équivalents le jeu est l'un de ces objets 
culturels pour lesquels l'équation « on fait ce que l'on aime, et l'on aime ce que l'on fait » ne peut fonctionner.

Au-delà des structurations de la pratique ludique en fonction de l'âge, ou du sexe, il importe donc de changer d'échelle pour considérer de plus près les pratiques telles qu'elles prennent place et sens dans le quotidien des joueurs. Sur la base de nos données qualitatives, nous montrerons ci-après que la pratique est d'abord inscrite dans des réseaux de sociabilités ; l'appétence, le goût pour l'objet en soi - ses formes, ses règles, son esthétique - importe moins que la situation. Aux relations à d'autres personnes s'ajoute la prise en compte des compétences techniques et ludiques du joueur, qui viennent complexifier davantage la relation entre goût et pratique.

\section{Des formes de sociabilité avant le goût}

Goût et pratique ne coïncident donc pas nécessairement. Cela ne signifie pas que nos enquêtés jouent à des jeux qu'ils n'aiment pas, mais simplement que leurs jeux favoris ne peuvent être pratiqués que dans certaines situations, quand d'autres invitent à jouer à des jeux moins enthousiasmants, mais plus adaptés. C'est par exemple le cas des soirées où Céleste ( 28 ans, gendarme, en couple, sans enfants) et son ami proposent à leurs invités tantôt des party games sur la console Wii, tantôt des jeux de société comme Time's Up. Lorsqu'ils jouent seuls ou en couple, à l'inverse, ils déclarent une préférence pour les jeux vidéo de rôle (Skyrim, Zelda) ou de foot $\left(P E S^{4}\right)$. Leur goût va aux seconds jeux, mais les premiers constituent une manière de jouer ensemble avec les invités. Cette pratique du jeu inscrite dans une situation de sociabilité est loin d'être anecdotique. Elle permet d'expliquer l'importance quantitative du jeu de construction chez les adultes ayant des enfants sans faire appel à l'hypothèse d'un goût prononcé et répandu pour ce genre - c'est l'accompagnement des enfants qui est premier.

Nos enquêtés témoignent de la nécessité de trouver un équilibre entre les jeux de compromis et les jeux pour lesquels ils ont de l'appétence, qu'ils rapportent aux situations de jeu. Le jeu doit s'adapter aux partenaires en présence. Il en ressort que, en situation, le type de jeu et le degré d'engagement peuvent varier rapidement avec la configuration sociale. Il suffit qu'un joueur intègre ou quitte le groupe pour basculer du jeu de compromis, sans appétence particulière, au jeu passionné.

Ben on joue parfois à Time's Up, mais j'aime pas forcément. [...] le Time's Up c'est avec [ma femme], avec sa sœur, son beau-frère, le cousin, c'est pour Noël en gros on se fait le réveillon de Noël et puis quand tout le monde s'en va, nous on reste jusqu'à 5-6 heures du matin et on se fait notre [soirée jeux]. (Claude, $\mathrm{H}$, 36 ans, Gestionnaire technique en immobilier, en couple, deux enfants)

4 Les party games sont des jeux vidéo conviviaux, à plusieurs joueurs, permettant des parties courtes et présentant des règles simples ; ils se prêtent donc à l'activité collective entre joueurs de niveaux différents. Les jeux vidéo de rôle s'inscrivent dans la durée - une partie dure plusieurs dizaines d'heures, que le joueur peut interrompre - ; il est difficile de commencer en cours de partie. Dans les jeux de sports, les différences de niveau des joueurs se font fortement ressentir. 
Ici, les deux formes de jeux peuvent s'enchaîner durant la même soirée, à la faveur de la modification du groupe (« quand tout le monde s'en va »). Claude, par ailleurs, apprécie les jeux de cartes ( Je fais des concours avec un ami, parfois on se fait une petite soirée belote »). L'opposition entre jeux avec appétence et jeux de compromis recoupe largement celle entre cercle amical et cercle familial, mais peut également traverser ce dernier.

Une telle situation est fréquente. Les entretiens révèlent, souvent entre les lignes, tout un volet de pratiques ludiques investies malgré un intérêt limité, voire absent. Que les enquêtés se considèrent comme joueurs ou non, on constate de manière générale la présence de ces modalités de jeu non passionnées dans leur quotidien familial. Mireille (F, 38 ans, chargée d'étude, mariée, deux enfants) par exemple raconte que chez elle enfant, en famille on jouait certes à «quelques jeux de société : La Bonne Paye, Monopoly»; elle s'empresse cependant de préciser: "Mais pas beaucoup. Enfin, c'était les vacances. C'était certains week-ends. Mais non il n'y a pas une tradition de jeu chez moi finalement. » Cela contraste avec les pratiques ludiques de son mari qu'elle qualifie de «vrai joueur». L'absence d'une véritable «tradition de jeu» n'implique pas l'absence de jeu, mais donne lieu à des pratiques investies selon des modalités distinctes, dans les discours des enquêtés, de celles pratiquées par les «vrais joueurs » ou dans le cadre d'une tradition familiale revendiquée. Des modalités n'impliquant pas un goût personnel, ou laissant celui-ci au second plan.

\section{Un jeu ancré dans des situations familiales}

Les pratiques ludiques familiales présentent malgré leur caractère sporadique quelques régularités temporelles : le jeu tend à prendre place à des moments spécifiques, comme les vacances, le week-end, les jours de fête, ou encore le mercredi, moments de temps libre et de coprésence d'adultes et d'enfants. Pour autant, le jeu n'est pas automatiquement associé à ces moments. Il ne suffit pas d'être ensemble : il faut encore que l'occasion s'y prête, et notamment qu'elle n'offre pas d'alternatives plus valorisées que le jeu, et que des jeux soient présents.

Quand on est en vacances, de temps en temps bon je joue aux cartes, ou des jeux que les enfants peuvent avoir, mais ce n'est pas... Ce n'est pas un rituel. Ce n'est pas tout le temps. [...] Si on va en montagne et puis qu'il ne fait pas beau et qu'ils ont emporté des jeux, oui, on va faire des choses comme ça. (Chantal, F, 56 ans, enseignante retraitée, en couple, 3 enfants)

L'énumération que fait Chantal des conditions du jeu souligne que ces pratiques ludiques bénéficient d'un statut secondaire, qu'elles prennent place à défaut de mieux. Si les jeux pratiqués dans ces occasions sont souvent des jeux classiques de cartes et des jeux dits de société, il arrive que des jeux vidéo aient la même fonction. Cela confirme notre hypothèse selon laquelle, moins que la nature technique des jeux, c'est leur inscription dans des configurations sociales qui importe. Laurence (F, 32 ans, coordinatrice dans une association, en couple, 2 enfants) joue parfois sur son iPhone avec ses enfants de 2 et 4 ans. Bien qu'elle souligne par ailleurs sa désapprobation pour l'usage excessif et solitaire des jeux vidéo chez les enfants d'autrui, elle glisse : « quand tout d'un coup tu as oublié le livre ou les crayons de couleur au restaurant, j'ai au moins deux ou trois petits jeux pour les enfants ». Chantal a des enfants adolescents et 
mentionne les occasionnelles sessions de jeu vidéo en famille, non sans préciser qu'elle ne partage pas par ailleurs leurs goûts en la matière : «Quand un jeu vient de sortir, et qu'on est en vacances tous ensemble, c'est vrai qu'on peut essayer de s'amuser à qui fera le meilleur score $»$.

Cependant, plus que les jeux classiques, les jeux vidéo tendent à favoriser un engagement déséquilibré des membres de la famille, les adultes prenant un rôle d'accompagnateurs, détachés de la pratique elle-même, et les enfants étant les véritables joueurs. Élise (F, 30 ans, gestionnaire, en couple, 1 enfant) se souvient de moments de jeu sur ordinateur avec sa mère à ses côtés, bien qu'elle juge ses parents «peu joueurs » (« Non je m'imagine mal mettre mes parents devant un jeu vidéo ») : «Je ne sais pas si ça compte vraiment ». La difficulté à qualifier la situation de jeu renvoie au peu de pertinence que revêt le jeu non passionné : ce n'est pas vraiment jouer ensemble que de jouer avec un enfant, du moins par opposition aux pratiques entre pairs. Laurence évoque également les «jeux pour les enfants » auxquels elle a accès sur son téléphone. Contrairement aux jeux de société, ou plus généralement aux jeux classiques, devant un écran on fait davantage jouer son enfant qu'on ne joue avec son enfant. Ces pratiques ludiques sont davantage centrées sur le plaisir et les besoins d'autrui que les siens propres. L'inverse est également vrai : la participation des enfants au jeu des adultes peut être limité à l'observation plus ou moins distante. Micka ( H, 32 ans, informaticien indépendant, en couple, sans enfant) évoque ainsi les parties de Trivial Pursuit durant lesquelles «ma petite sœur et moi, on devait plutôt jouer en support, je veux dire, en regardant ».

\section{Des goûts de compromis}

Si nous parlons, pour désigner ces situations, de jeu «sans appétence », l'expression ne doit pas être interprétée comme la négation du plaisir : rien n'exclut que des pratiques ludiques, investies dans un registre qui n'est pas celui du goût, aient aussi pour résultat, attendu ou non, un moment plaisant pour ceux qui s'y adonnent. Ce n'est donc pas tant l'absence, mais plutôt la relégation au second rang du goût individuel et des préférences esthétiques qui est à l'œuvre. On le remarque en particulier dans la faible présence du goût dans le registre de justification des acteurs. Alors que la plupart des pratiques culturelles font d'abord l'objet d'une justification par «l'amour de l'art », nos enquêtés n'utilisent que très rarement ce vocabulaire.

Le goût est secondaire en particulier dans le choix des jeux. C'est la nécessité de trouver un compromis entre les goûts et les capacités des différents participants, d'une part, et les opportunités offertes par la situation d'autre part qui détermine le jeu qui sera pratiqué. Amélie (F, 29 ans, enseignante, en couple, sans enfants) explique ainsi le choix du Memory du mercredi après-midi, qu'elle partageait avec sa grand-mère et ses cousins : "On avait quand même des écarts d'âge importants donc du coup pour des jeux qui intéressaient tout le monde c'était compliqué. » Le compromis se fait souvent au profit du plus jeune, lorsque le jeu est une activité à destination des enfants. Il se fait parfois au profit des adultes ou des adolescents, comme dans le cas du Trivial Pursuit que raconte Micka plus haut.

Même là où il y a peu d'appétences, il faut ménager les diverses capacités des participants à s'investir. Une explication pour l'évolution différentielle entre pratiques 
des jeux vidéo et classiques au fil de l'âge s'esquisse sur cette base : jouer en famille à des jeux vidéo semble ainsi plus compliqué en termes de consensus que de choisir un jeu classique, parce que le jeu vidéo s'adresse plus spécifiquement à certaines générations, et parce qu'il demande des compétences plus spécifiques que le jeu traditionnel. Alors que nombre de jeux traditionnels sont fondés sur le simple hasard ou sur des compétences cognitives générales (logique, calcul, mémoire), le jeu vidéo exige au moins une certaine aisance manuelle (manipuler le terminal), ainsi que, le plus souvent des notions de stratégies propre au monde des jeux vidéo. C'est ce que confirme ici Céleste, à propos des tentatives de jouer à Mario Kart avec sa sœur et ses parents : «C'est vrai que ce n'est pas du tout leur génération [...] Ils l'ont fait pour nous faire plaisir une ou deux fois, mais ça s'est arrêté là ».

Le jeu sans appétence, enfin, n'est pas nécessairement un jeu sans sélectivité. Les ajustements et compromis mentionnés ne sont pas toujours possibles. De fait, nombre de jeux voient participer des joueurs qui ne les apprécient pas particulièrement. Pour autant, on ne joue pas à n'importe quoi avec n'importe qui. Les entretiens montrent des régularités dans les lieux et moments du jeu, mais aussi dans les participants. Ces régularités, sans constituer des goûts très affirmés, se muent avec le temps en systèmes de préférences faiblement affirmées. Micka explique que le Monopoly «ça, c'était le jeu de famille avec mon grand-père et mon oncle, en vacances », en revanche «le Trivial Pursuit, ça, c'est plutôt avec mon père ». Ou encore Étienne, chez qui il y a eu un partage entre le jeu de l'oie avec sa mère et la belote avec ses grands-parents; avec son père par contre, il ne jouait pas car « il n'aimait pas ça ». Même parmi les pratiques familiales donc, il y a sinon une électivité, au moins une sélectivité. Des logiques de choix, tant du joueur que du jeu adéquat, sont à l'œuvre.

Partant des résultats statistiques sur les différences entre jeu vidéo et non-vidéo, nous avons ici tenté d'esquisser les contours d'une modalité de jeu décrite comme peu passionnée ou sans appétence et dont on peut faire l'hypothèse qu'elle sous-tend une bonne partie des pratiques ludiques classiques répandues dans la population, et en particulier celles prenant place dans le cercle familial avec enfants. Il est en même temps apparu qu'il ne s'agit pas d'une modalité exclusive : elle n'est propre ni aux relations intergénérationnelles - on l'aperçoit dans la fratrie ou entre cousins par exemple -, ni aux jeux classiques, car certains jeux sur écran s'y prêtent également. Nous en concluons que, plutôt que des propriétés essentielles de l'une et l'autre catégorie, ce qui différencie au niveau les pratiques de jeux classiques et celles des jeux vidéo, c'est le type de sociabilité dans lesquelles ces pratiques s'ancrent. Les modalités de pratique des jeux classiques relèvent davantage de l'habitude familiale, investies sur un registre sans appétence.

\section{Quand le goût fait la pratique}

Il serait évidemment réducteur de considérer l'ensemble des pratiques ludiques adultes comme dénuées d'appétence. Certaines ne s'inscrivent pas dans des sociabilités familiales, mais constituent de véritables pratiques passionnées. Ce sont d'abord les jeux vidéo, dont la pratique intensive persiste parfois à l'âge adulte, mais aussi certains jeux classiques faisant l'objet d'un engagement important, ancré dans des sociabilités électives : pratique compétitive ou amicale du jeu de cartes, jeux de société complexes, 
etc. Ces pratiques sont les plus visibles dans la littérature de sciences sociales : les joueurs d'échecs (Wendling 2002), les amateurs de jeux de rôle (Fine 1983; Trémel 2001), les passionnés de jeux vidéo (Taylor 2006) ont été largement étudiés. Nous avons privilégié jusqu'ici l'analyse des pratiques moins passionnées, mais la distinction entre pratiques passionnées et pratiques ordinaires mérite qu'on s'y arrête dans cette dernière section pour éclairer leurs places respectives parmi les pratiques ludiques. Nous revenons enfin sur la coexistence, chez les mêmes individus, de ces pratiques engagées avec des pratiques plus nonchalantes, ainsi que sur leur inscription dans des univers culturels plus larges.

\section{Les pratiques "fans ॥ révélatrices de hiérarchies ludiques et de sociabilités sélectives}

Les jeux classiques qui font l'objet de passion sont souvent opposés par les enquêtés aux jeux «simples »; ils se définissent ainsi en creux, par opposition aux jeux trop consensuels, par leur complexité. Ils nécessitent des équipements nombreux, la lecture et la compréhension de règles difficiles, la mise au point de stratégies élaborées, voire même, souvent, la connaissance tacite d'autres univers fictionnels. Ces propriétés apparaissent dans les propos de $\operatorname{Marc}(\mathrm{H}, 33$ ans, enseignant, vit seul, sans enfant) : temps de la partie, complexité des règles, complexité des stratégies et hermétisme des références. Marc raconte avoir eu une compagne avec qui il ne parvenait pas à partager son goût pour un jeu de société complexe, inspiré par l'univers fictionnel de l'écrivain fantastique H. P. Lovecraft : " C'est d'abord toute une littérature de Lovecraft. Il y a des références qui partent. On sait à peu près d'où viennent des monstres. On sait de quelle histoire c'est tiré. » Et étant donné que les parties de son jeu durent plusieurs heures, sa tentative de faire jouer son amie «n'a pas été un grand succès [...] Pour nous c'est un vrai plaisir, mais pour certains c'est... Ça peut demander beaucoup. »

La distinction entre jeux complexes et simples est également partagée par les nonspécialistes ; pas besoin d'en être pour reconnaître qu'il y a une différence. Chantal (F, 56 ans, enseignante retraitée, en couple, 3 enfants) commente les jeux vidéo que pratiquent ses enfants en mobilisant cette opposition. Elle mentionne les jeux de plateforme qu'elle qualifie de « débiles » parce qu'il n'y a " pas trop de recherche làdedans » ou encore " des jeux bateaux comme Sudoku et les jeux de cartes [...] je ne vois pas trop l'interactivité », jeux qu'elle oppose à Assassin's Creed, un jeu d'actionaventure, qui comporte «des étapes, des choses à faire pour aller d'un endroit à un autre », où il y a «un rôle à jouer ». Chantal identifie des propriétés ludiques de complexité et d'immersion du joueur dans un rôle. Ce faisant, elle rejoint la valorisation de la dimension narrative du jeu chez les joueurs férus de jeux de rôle. Le goût narratif que tendent à développer ces joueurs se retrouve dans leur manière de jouer tant aux jeux vidéo que de société. Par exemple Georges $(\mathrm{H}, 32$ ans, enseignant, en couple, sans enfant) qui joue depuis longtemps et quotidiennement sur console fixe et portable, PC et plus récemment sur smartphone avec une préférence pour les jeux d'aventure et de rôle, organise aussi des soirées de jeux de société entre amis. Parmi ceux-là, il affectionne particulièrement des jeux de rôle sur plateau, coopératifs, avec un matériel relativement riche en pièces et en textes, des jeux aux mondes fictifs souvent tirés de séries télévisées ou de livres. Il explique que pour lui et les amis avec qui il joue, «l'histoire est importante », ce qui façonne le déroulement de leurs parties de jeux de société : «on lit 
chacun l'histoire [...] donc quand on découvre un jeu on met toujours deux heures histoire que tout le monde soit au courant. »

Les engagements que décrivent Georges et Marc peinent à prendre place dans les sociabilités astreintes. Le jeu que cite Marc ne fait pas l'objet de parties en famille ou même entre amis si ceux-ci n'ont pas une passion pour le jeu. Il ne peut s'inscrire que dans des sociabilités électives, dans le cadre d'un groupe de passionnés, qui organisent leurs rencontres autour du jeu. Dans ce cas, la pratique du jeu est liée à l'intérêt pour l'univers fictionnel dans lequel il prend place, rejoignant le phénomène des cultures « fan » qui tendent à consommer et à s'investir en parallèle dans divers supports médiatiques, des jeux vidéo à la littérature, en passant par le cinéma et les séries TV (Jenkins 1992).

Ce lien entre le jeu et les autres intérêts culturels des individus s'observe fréquemment dans les pratiques engagées. Si les pratiques sans appétences décrites dans la section précédente se caractérisent le plus souvent par leur inscription dans une culture partagée, le jeu autonome appartient plus souvent à des sous-cultures plus clairement délimitées. Vincent Berry montre par exemple les liens qui existent entre la pratique des jeux de rôle en ligne, la musique métal, et la littérature et le cinéma de fantasy (Berry 2012). Mais ces univers culturels très spécifiques ne sont pas les seuls à accueillir des pratiques de jeu passionné. L'intérêt pour le sport, par exemple, est aussi susceptible d'alimenter des engagements ludiques. Cela se donne à voir chez Lionel $(\mathrm{H}$, 39 ans, chef de cuisine, en couple, deux enfants), qui aime la course automobile, fait partie d'un fan-club d'Alain Prost et raconte sa pratique du jeu de console Formula One : c'est «le seul jeu où vraiment je crois que j'ai été jusqu'au bout. Je l'ai terminé. J'ai fait tous les circuits, et tout. Et c'est le seul jeu où j'essayais vraiment d'être le meilleur. » La pratique vidéoludique de Lionel s'inscrit dans sa passion ordinaire plus large, tout comme la pratique du jeu de société s'inscrit dans les pratiques «fan» de Georges et Marc.

Nous ne prétendons pas, avec ces quelques cas, épuiser les possibilités du jeu des adultes, mais seulement souligner que des pratiques relativement autonomes des sociabilités familiales peuvent exister. Elles demandent cependant des arrangements particuliers pour pouvoir persister, comme le maintien d'une sociabilité avec d'autres passionnés - amis, collègues de bureau -, ou encore l'organisation du temps consacré à la famille. Il faut souligner que même les pratiques les plus passionnées ne sont pas dénuées de contraintes. C'est ce que montre cette dernière section.

\section{Jeux passionnés sous contraintes}

On l'aura deviné au vu des effets de variables démographiques et de configuration familiale sur les jeux pratiqués: les pratiques ludiques, même à fort degré d'engagement, ne sont pas qu'une affaire de passion. Les récits de vie d'enquêtés donnent à voir comment les joueurs perçoivent les effets de leur mise en couple, l'arrivée de leur enfant, ou encore l'entrée dans la vie professionnelle sur leurs pratiques ludiques. Les enquêtés se remémorent fréquemment des pratiques ludiques plus intenses avant de tels évènements biographiques, allant parfois jusqu'à oublier que leurs pratiques actuelles sont à certains égards tout aussi conséquentes, mais suivant de 
nouvelles modalités, typiquement sur téléphone portable ou tablette à la place de la console ou du PC.

Je ne joue plus ni même sur PC, ni Mac. [...] J'avais un peu arrêté, et finalement le support de la tablette, avec un autre style de jeu, en fait très court avec une durée de vie très limitée, très simple à prendre en main etc. m'a fait me remettre un peu au jeu. (Guy, H, 33 ans, cadre supérieur de l'administration, en couple, 2 enfants)

Les récits de ce type de modification des styles ludiques sont courants dans nos entretiens. Dans des cas plus exceptionnels, les joueurs assidus ont redoublé d'efforts pour maintenir des pratiques ludiques de passionnés, comme Michel (H, 34 ans, informaticien, en couple sans enfants) qui explique avoir trois façons de jouer : quotidiennement sur le téléphone mobile dans les transports en commun, à la maison, mais seul sur son ordinateur (où il pratique des jeux " pour joueurs ", c'est-à-dire complexes) et à la maison sur la console du salon, avec sa femme. Cette dernière modalité leur permet d'entretenir un goût commun pour les «jeux à licence qu'on aime bien » comme les jeux de super héros de la franchise Lego, qui se jouent parfois en coopératif ou alors en solo avec l'autre partenaire en spectateur. Ses pratiques de « jeux pour joueurs » sont réservées à des moments bien précis, à l'heure de la sieste de sa compagne. Ainsi, Michel concilie sa pratique passionnée et la vie de couple, partageant une partie de sa passion.

Les modalités du jeu passionné, et en particulier les pratiques de jeu vidéo, font apparaitre et se confronter deux types de normes. D'une part une hiérarchie de la valeur du jeu, les « vrais » jeux, complexes, et qui requièrent et des sociabilités sélectives, et les jeux plus "simples ». Mais en parallèle à ces gradations de la qualité ludique se dessinent des jugements sur les formes d'engagement dans le jeu. L'exemple de Michel montre que, comme nous le décrivons plus en détail ailleurs (Gerber, 2015), les pratiques ludiques sont négociées autour d'attentes de rôles ancrées dans les sociabilités.

\section{Conclusion}

Si nous avons distingué dans cet article les pratiques ludiques classiques de celles des jeux vidéo, il importe de ne pas réifier cette opposition, et notamment de ne pas la rabattre sur la technique numérique. Il s'agit de deux ensembles hétérogènes, certains genres de jeu vidéo se rapprochant des jeux classiques, et certains genres classiques permettant le même engagement que le jeu vidéo. La coupure entre jeux «simples » et « complexes »-par le degré et la forme des compétences exigées par le jeu-, jeux «pour joueurs » et jeux familiaux, apparaît bien plus pertinente. Elle permet d'ancrer les pratiques ludiques dans les configurations de sociabilités familiales et amicales. Ainsi, durant l'enfance, la pratique du jeu vidéo diffère peu de celle du jeu classique, alors qu'elle s'en déconnecte largement à l'adolescence.

Cela permet de comprendre pourquoi les jeux vidéo divisent les générations, en participant à autonomiser la culture jeune, alors que les jeux classiques tendent à les rassembler, de par leur pratique en famille. L'effet de la génération sur les pratiques ludiques doit alors être qualifié. On peut certes penser que, dans les prochaines 
décennies, la banalisation de l'usage du jeu vidéo sera telle qu'il aura intégré le répertoire des jeux classiques qui aliment encore aujourd'hui les sociabilités familiales. Pour autant, la complexité de certains jeux n'aura sans doute pas disparu, et de nouvelles techniques pourront produire de nouvelles distinctions. Notre recherche permet donc de mettre en lumière les mécanismes de la coupure ludique générationnelle : elle est à chercher dans les réseaux de sociabilités valorisés par les différentes pratiques.

Les pratiques ludiques constituent, au-delà, un terrain idéal pour saisir les tensions qui existent entre goûts et pratiques en matière de loisirs et de culture. On y voit mieux qu'ailleurs que l'appétence n'est pas nécessairement la cause première de la pratique, et surtout est rarement pensée comme telle. Le jeu obéit à bien d'autres logiques : la présence d'occasions, de dispositifs matériels, de formes sociales adaptées notamment. Il a, comme la photographie, une fonction familiale et pas seulement une fonction esthétique (Bourdieu 1965). Il importe alors saisir les pratiques en tant qu'elles sont insérées dans les biographies des joueurs, ainsi que dans leurs écosystèmes.

Une telle conclusion ne devrait pas être arrêtée aux seuls jeux. Si nous voulons, par ce travail, inciter les sociologues de la culture comme ceux de la famille à s'intéresser à cet objet, c'est en tant qu'il donne à voir des questions qui se posent dans d'autres situations. Celle des liens entre pratique et appétence nous paraît particulièrement centrale dans la sociologie de la culture contemporaine. Les sociologues considèrent habituellement les deux comme interchangeables : tantôt la pratique déclarée est une approximation du goût, tantôt le goût déclaré est une approximation de la pratique (pour une réflexion critique, cf. Robette et Roueff 2014). Dans le cas des consommations culturelles ordinaires, cette idée est d'autant plus ancrée qu'elle s'accorde avec le sens commun. Pourtant, les consommations culturelles modernes sont pleines de goûts partiellement assouvis et de pratiques contraintes ou produites par la situation. Le passage par les sociabilités est alors une manière de rendre compte de ce décalage entre goût et pratique (Dubois et al. 2010 ; Pasquier 2005a).

Ce décalage ne signifie pas que le goût soit absent des pratiques de jeu. Simplement, il n'est pas premier dans les choix opérés. Il faut accorder les goûts et les capacités de l'ensemble des participants, et s'adapter à la situation. En conséquence, le jeu choisi peut n'être le favori de personne. Il peut même, dans certains cas, être l'objet d'une perception négative par la quasi-totalité des participants. Une enquête inédite sur la pratique du Monopoly semble suggérer qu'il s'agit d'un cas typique de compromis familial: sa pratique persiste dans certaines familles sans que ni les parents ni les enfants n'aiment le jeu (Vincent Berry, communication personnelle). Le plaisir n'est pourtant pas absent du jeu, ne serait-ce que parce qu'il est pris simplement dans l'interaction que permet celui-ci. Le jeu invite alors à ne pas considérer, dans l'analyse des consommations culturelles, le goût pour l'objet consommé comme étant le seul moteur de la consommation.

\section{Références}

Berry, Vincent. 2012. L'expérience virtuelle. Jouer, vivre, apprendre dans un jeu vidéo, Rennes Presses Universitaires de Rennes. 
Bickel, Jean-François, Christian Lalive d'Épinay et Nathalie Vollenwyder. 2005. «Changements et continuités dans les loisirs. Une comparaison de cohortes ", L'année sociologique, vol. $55, \mathrm{n}^{\circ} 1$, pp. 129-169.

Bordes, Pierre. 2009. «La sportification: ses mécanismes et ses effets ». Congrès de l'Association française de sociologie.

Borgers, Natacha, Edith de Leeuw et Joop Hox. 2000. «Children as respondents in survey research. Cognitive development and response quality », Bulletin de méthodologie sociologique, ${ }^{\circ}$ 66, pp. 60-75.

Borzakian, Manouk. 2012. «Les jeux: quelle définition par et pour les sciences sociales? », Loisir et société/Society and Leisure vol. 35, $\mathrm{n}^{\circ} 2$, pp. 341-60

Borzakian, Manouk. 2015. «Une géographie de la France ludique. La dimension spatiale des pratiques culturelles » Annales de géographie, 701, pp. 51-75.

Boullier, Dominique. 2004. «La fabrique de l'opinion publique dans les conversations télé », Réseaux, vol. $126, \mathrm{n}^{\circ} 4$, pp. 57-87.

Bourdieu, Pierre. 1965. Un art moyen. Essai sur les usages sociaux de la photographie, Paris, Minuit.

Boutet, Manuel. 2008. «S'orienter dans les espaces sociaux en ligne. L'exemple d'un jeu », Sociologie du travail, vol. 50, $\mathrm{n}^{\circ} 4$, pp. 447-470.

Boutet, Manuel. 2003. «Des jeux d'adultes? Corporéités et sociabilités dans les cyberespaces ludiques » in La pratique du jeu vidéo: Réalité ou virtualité, Mélanie Roustan (dir.), Paris, L'Harmattan, pp. 99111.

Boutet, Manuel, Isabel Colon de Carvajal, Hovig Ter Minassian et Mathieu Triclot. 2014. « Au-delà du virtuel : interactions sociales et spatiales dans et autour d'un univers vidéoludique », Médiation et Information, $\mathrm{n}^{\circ} 37$, pp. 103-115.

Brougère, Gilles. 2013. «Jacques Henriot et les sciences du jeu ou la pensée de Villetaneuse », Sciences du jeu, vol. 1, http://sdj.revues.org/202.

Brousse, Cécile. 2015. « Travail professionnel, tâches domestiques, temps 'libre'. Quelques déterminants sociaux de la vie quotidienne », Economie et statistique, n 478-479-480, pp. 119-154.

Cardon, Dominique et Fabien Granjon. 2003. «Éléments pour une approche des pratiques culturelles par les réseaux de sociabilité », in Olivier Donnat et Paul Tolila (dir.) Le(s) public(s) de la culture, Paris, Presses de Sciences Po.

Cézard, Michel, Michel Gollac et Catherine Rougerie. 2000. «L'ordinateur, outil de travail et bien culturel », Actes de la recherche en sciences sociales, $\mathrm{n}^{\circ} 134$, pp. 22-28.

Chenu, Alain et Nicolas Herpin. 2002. «Une pause dans la marche vers la civilisation des loisirs ? », Économie et Statistique, n 352-353, pp. 15-37.

Coulangeon, Philippe, Pierre-Michel Menger et Ionela Roharik. 2002. « Les loisirs des actifs : un reflet de la stratification sociale », Économie et Statistique, vol. 352, n $1: 39-55$.

Dajez, Frédéric et Nathalie Roucous. 2010. «Le jeu vidéo, une affaire d'enfants. Enquête sur le parc à jouets numérique d'enfants de 6 à 11 ans », in Sylvie Octobre (dir.) Enfance et culture, Paris, DEPS, pp. 83-101.

Dajez, Frédéric et Nathalie Roucous. 2009. «Montre-moi tes jeux vidéo ! », Mutations, n 1, pp. 74-91.

Dayan, Daniel. 1986. «Copyrighted Subculture», American Journal of Sociology, vol. 91, pp. 1219-1228.

Degenne, Alain et Marie-Odile Lebeaux. 2003. «Le temps des loisirs, le cycle et ses contraintes », in Olivier Donnat (dir.) Regards croisés sur les pratiques culturelles, Paris, La documentation française : 83-105.

DeNora, Tia. 2001. «Quand la musique de fond entre en action », Terrain, ${ }^{\circ}$ 37, pp. 75-88.

Digard, Jean-Pierre. 2001. «Les courses de chevaux en France », Études rurales, n 1, pp. 95-106. 
Donnat, Olivier. 2009. Les pratiques culturelles des français à l'ére numérique. Enquête 2008, Paris, La Découverte.

Dubois, Vincent, Jean-Matthieu Méon et Emmanuel Pierru. 2010. «Quand le goût ne fait pas la pratique », Actes de la recherche en sciences sociales, vol. 181-182, n 1, pp. 106-125.

Duflo, Colas. 2001. «Grandeur et misère du jeu à l'ère du divertissement ». Cités, vol. 7, n 3, pp. 109-118.

Dumazedier, Joffre. 1962. Vers une civilisation du loisir?, Paris, Seuil.

Elias, Norbert et Eric Dunning. 1986. Quest for excitement: Sport and leisure in the civilizing process. Oxford, Blackwell.

Fine, Gary Alan. 2015. « Organiser les mondes de loisir. La mobilisation des ressources », Tracés, no 28.

Fine, Gary Alan. 2012. "Time to Play. The Temporal Organization of Chess Competition », Time and Society, vol. 21, $\mathrm{n}^{\circ} 3: 395-416$.

Fine, Gary Alan. 1983. Shared Fantasy : Role-Playing Games as Social Worlds, Chicago, University of Chicago Press.

Forsé, Michel. 1981. « La sociabilité », Economie et statistique, vol. 132, pp. 39-48.

Gerber, David. 2015. «Le jeu vidéo comme pratique discréditable. Enquête sur les efforts de légitimation ordinaire des joueurs ", RESET, vol. 4. http://reset.revues.org/452

Gire, Fabienne, Dominique Pasquier et Fabien Granjon. 2007. «Culture et sociabilité. Les pratiques de loisir des français », Réseaux, vol. 145-146, n 5, pp. 159-215.

Granjon, Fabien et Aurélien Le Foulgoc. 2010. «Les usages sociaux de l'actualité : L'Expérience médiatique des publics internautes », Réseaux, vol. 160, n² 2, pp. 225-253.

Harrington, Maureen. 2006. «Family Leisure », in Chris Rojek, Anthony J. Veal et Susan M. Shaw (dir.) Handbook of Leisure Studies, Londres, Palgrave Macmillan, pp. 417-432.

Henricks, Thomas. 2010. «Play and Cultural Transformation - Or, What Would Huizinga Think of Video Games? » in T. J. Wright (dir.) Utopic Dreams and Apocalyptic Fantasies: Critical Approaches to Researching Video Game Play, Lexington Books, pp. 15-42

Huizinga, Johan. 1977. Homo ludens: essai sur la fonction sociale du jeu. Paris, Gallimard.

Jenkins, Henry. 1992. Textual Poachers. Television Fans and Participatory Culture, Londres, Routledge.

Kelly, John R. 1995. "Leisure and the Family», in Chas Crichter, Peter Bramham et Alan Tomlinson (dir.) Sociology of Leisure. A Reader, New York, Taylor \& Francis, pp. 44-54.

Lahire, Bernard. 2004. La culture des individus. Dissonances culturelles et distinction de soi, Paris, La Découverte.

Le Douarin, Laurence. 2007. «Les chemins de l'articulation entre vie privée et vie professionnelle », Réseaux, $\mathrm{n}^{\circ} 1$, pp. 101-132.

Lin, Holin. 2014. «An Entertainment Machine of Filial Piety. A Look at Wii as a Gift for Parents », Reset, $\mathrm{n}^{\circ} 3$, http://reset.revues.org/389.

Lizardo, Omar. 2006. «How Cultural Tastes Shape Personal Networks », American Sociological Review, vol. $73, \mathrm{n}^{\circ} 5$, pp. $697-718$.

Lizé, Wenceslas. 2004. «Imaginaire masculin et identité sexuelle », Sociétés contemporaines, $\mathrm{n}^{\circ} 3$, pp. 43-67.

Martignoni-Hutin, Jean-Pierre. 1997. «Les jeux de hasard. Faire de l'argent et faire avec l'existence », Agora Débats Jeunesses, vol. 10, n $1: 49-60$.

Martin, Olivier. 2004. «L'Internet des 10-20 ans. Une ressource pour une communication autonome », Réseaux, $\mathrm{n}^{\circ} 123$, pp. 25-58. 
Mood, Carina (2010). « Logistic Regression. Why We Cannot Do What We Think We Can Do, and What We Can Do About It », European Sociological Review, vol. 26, n 1, pp. 67-82.

Nowak, Raphaël et Andy Bennet. 2014. «Analysing Everyday Sound Environments. The Space, Time and Corporality of Musical Listening », Cultural Sociology, vol. 8, n 4, pp. 426-442.

Octobre, Sylvie, Christine Détrez, Pierre Mercklé et Nathalie Berthomier. 2010. L'enfance des loisirs : Trajectoires communes et parcours individuels de la fin de l'enfance à la grande adolescence, Paris, La Documentation Française.

Pasquier, Dominique. 2012. «La sortie au théâtre. Réseaux de conseil et modes d'accompagnement », Sociologie, vol. 3, $\mathrm{n}^{\circ} 1$, pp. 21-37.

Pasquier, Dominique. 2005a. Cultures lycéennes. La tyrannie de la majorité, Paris, Autrement.

Pasquier, Dominique. 2005b. « La « culture populaire » à l'épreuve des débats sociologiques ». Hermès $\mathrm{n}^{\circ} 42$, pp.60-69.

Pearce, Celia. 2009. Communities of Play: Emergent Cultures in Online Games and Virtual Worlds, Cambridge, MIT Press.

Pharabod, Anne-Sylvie. 2004. «Territoires et seuils de l'intimité familiale. Un regard ethnographique sur les objets multimédias et leurs usages dans quelques foyers franciliens », Réseaux, ${ }^{\circ}$ 123, pp. 85-117.

Robette, Nicolas et Olivier Roueff. 2014. «An eclectic eclecticism: Methodological and theoretical issues in the quantification of cultural omnivorism ", Poetics, vol. 47, pp. 23-40.

Rufat, Samuel, Hovig Ter Minassian et Samuel Coavoux. 2014. «Jouer aux jeux vidéo en France. Géographie sociale d'une pratique culturelle », L'Espace géographique, vol. 43, n 4, pp. 308-323.

Scott, Jacqueline. 2000. «Children as Respondents. The Challenge for Quantitative Methods» in Pia Christensen et Allison James, Research with Children. Perspectives and Practices, Londres, Routledge, pp. 98-119.

Smoreda, Zbigniew. 2002. «Présentation. Cycles de vie et sociabilité », Réseaux, vol. 115, pp. 9-13.

Taylor, T. L. 2006. Play Between Worlds. Exploring Online Game Culture, MIT Press.

Trémel, Laurent. 2001. Jeux de rôles, jeux vidéo, multimédia. Les faiseurs de monde, Paris, Presses Universitaires de France.

Vincent, Sandrine. 2000. "Le jouet au cœur des stratégies familiales d'éducation », Sociétés contemporaines, vol. 40, pp. 165-182.

Vincent, Sandrine. 2001. «Le rôle du jouet dans la mémoire familiale ou comment les jouets finissent-ils leur vie ? » Dialogue, vol. 154, n 4, pp. 99-106.

Wendling, Thierry. 2002. Ethnologie des joueurs d'échecs, Paris, Presses Universitaires de France.

Winnicott, Donald W. 1971. Playing and reality. Burns \& Oates.

Yonnet, Paul. 1980. «Le tiercé, miroir social. Anthropologie d'un jeu de masse », Le Débat, vol. 6, pp. $45-55$

Yonnet, Paul. 1985. Jeux, modes et masses : la société française et le moderne 1945-1985. Paris, Gallimard. 